\title{
Computational Fluids Dynamics Analysis at First, Second and Third Hydrokinetics Turbine Generation
}

\author{
Cristian Cardona-Mancilla, Jorge Sierra-Del Rio* and Diego Hincapie-Zuluaga \\ Facultad de Ingenieria, Instituto Tecnologico Metropolitano. Medellin, Colombia; \\ cristiancardona@itm.edu.co, jorgesierra@itm.edu.co, diegohincapie@itm.edu.co
}

\begin{abstract}
Objectives: To increase the hydrodynamic efficiency of horizontal axis hydrokinetic turbines from the implementation of various geometric configurations of diffusers. Methods/Statistical Analysis: The turbine has a radius of $0.75 \mathrm{~m}$ and it's made up of three blades, they were designed using the hydrodynamic profile NREL S822. On the turbine a second and third generation diffuser are also implemented. The simulation was developed in the ANSYS CFX18.2 program in transitory regime, using a turbulence model $\mathrm{k}-\varepsilon$, a water input speed of $1.5 \mathrm{~m} \mathrm{~s}^{-1}$ and a variation of the angular velocity from 0 to 160 RPM. Include the method adapted to study the objectives/sampling details or simulation or statistical analysis of data; technique employed; mention unique/important points of modification of methodology in the current study. Mention about test samples the control employed or approach used for comparing the test sample. Findings: As a result, the power coefficient $\left(C_{p}\right)$ of the three generations of the hydrokinetic turbine was obtained, in addition to the velocity profile of each model. Increase in velocities both upstream and downstream of the turbine were found for the thirdgeneration turbine, presenting values of 1.5 to $2.1 \mathrm{~m} \mathrm{~s}^{-1}$ and 2.3 to $2.6 \mathrm{~m} \mathrm{~s}^{-1}$, respectively. The maximum power coefficient $\left(C_{p, \max }\right)$ reached by the first, second and third generation turbine was 0.285 at a TSR of $100,0.296$ at a TSR of 90 and 0.487 at a TSR of 95 , respectively. This is equivalent to an increase of $41.5 \%$ and $39.2 \%$ of the $C_{p}$ of the third-generation model with respect to the first and second-generation turbine, respectively and of $82.1 \%$ in relation to the Betz limit. Mention your findings in the form of statements along with the conclusive data of statistical importance; Mention how your findings are unique and novel; how your findings are in consensus with the existing values/reports or how different are they from the already reported findings. Highlight how your results are helpful in adding more value to the existing reports. Application/Improvements: The implementation of this type of turbines is aimed at the generation of electric power, promoting in greater measure the distributed generation in isolated areas near water sources.
\end{abstract}

Keywords: CFD, Diffuser, Hydropower, Kinetic, Renewable-Energy

\section{Introduction}

The hydrokinetic turbines emerge with the goal of using the kinetic energy in marine currents, rivers, artificial channels and others, without interrupting the water's natural flow, directly transforming it in mechanical power ${ }^{1}$. Their great reception is due in large part to the little infrastructural complexity they need for their operation, as they do not depend on large heights, presenting low costs and implementation times, as well as minimal environmental impacts with respect to conventional power plants $\frac{2-4}{4}$.

${ }^{*}$ Author for correspondence
The first horizontal axis hydrokinetic turbine was developed in 1987 by the research group of the Mechanical Department of the University of Brasilia (UnB), for which it received the qualification of first-generation hydrokinetic turbine. This mechanism, capable of generating $1.5 \mathrm{~kW}$ under operating conditions of currents with speeds of $2 \mathrm{~m} \mathrm{~s}^{-1}$, constituted by 2 blades and a diameter of $0.8 \mathrm{~m}$, was implemented to supply the electrical need of a medical post in Correntina, $\mathrm{BA}^{5}$. Under the premise that the main disadvantage of these devices compared to conventional hydroelectric power plants is the low density of electrical energy that they can supply, different 
studies and improvements were made in order to increase their efficiency and economic feasibility $\frac{3.6}{6}$. One of these improvements was the implementation of a conical diffuser, which was adapted from the results obtained in wind turbines, using the great similarity between both types of turbines. This device allows increasing the speed of the fluid upstream of the turbine, from the decrease of the pressure downstream of this, providing an increase in the kinetic energy of the fluid, being reflected in a greater efficiency. This development was classified as a secondgeneration hydrokinetic turbine. At present, investigations are done in order to improve the performance of these mechanisms, giving way to a third-generation hydrokinetic turbine. This new mechanism, formed by a profiled casing that surrounds the turbine, which fulfills the function of a first diffuser and a second cut-out diffuser coupled in serial form to the casing Figure 1 can reach hydraulic efficiencies close to $90 \%$ with respect to what is established by the Betz limit. This increase is caused by an existing opening at the radial level between the housing and the diffuser, which generates an increase in the energy of the fluid downstream of the turbine by conservation of time within the study's control volume, by allowing the passage of the flow from the outside to the inside of the mechanism, producing a drag force that helps to evacuate the fluid that passes through the turbine towards the outlet of the mechanism, reducing recirculation currents that are associated with energy losses in the fluid ${ }^{5}$.

A similar study to this new conception of turbine of third generation is made by ${ }^{\mathrm{T}}$, which had as main objective to design a Pico-electric power system of little complexity, economic and with the capacity of increasing the power of any hydrokinetic turbine, both horizontal (HAWT) and vertical (VAWT). Under this premise, they computationally designed and analyzed two mechanisms, the first of them with a rectangular throat section to house VAWT and the other with a circular section for HAWT, both composed of four components: 1. A convergent-divergent diffuser that adopted the shape of a Venturi nozzle described by the standard DIN EN ISO 5167-3: 2003, 2. A second diffuser surrounding the first device, 3 . The establishment of a flange $\mathrm{S}$ at the outlet of the second diffuser and 4 . A divergent internal diffuser downstream of the turbine. The integration of these improvements allowed increasing in 2.25 and 2.33 times the speed of the flow for the first and second models, respectively, representing for them an increase of 11.4 and 12.7 times their power output.

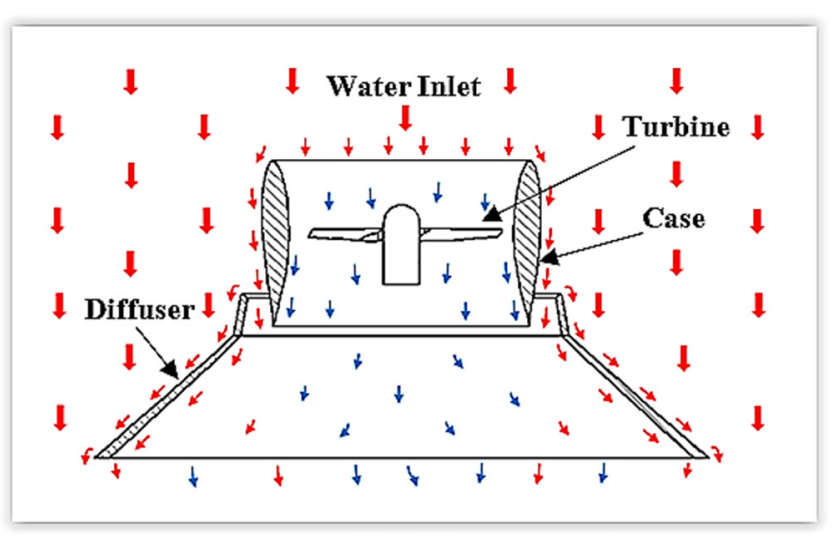

Figure 1. Hydrodynamic design of a hydrokinetic turbine with horizontal axis and third generation diffuser. Own source.

The objective of this study is to perform the hydrodynamic analysis by computational simulation of a hydrokinetic turbine with horizontal axis of the first, second and third generation, which seeks to increase the efficiency of this type of technology and promote its use as a source of renewable energy.

\section{Theory}

Hydrokinetic turbines and wind turbines have a very similar design philosophy because they operate under the same operating principles. The design of hydrokinetic turbines with horizontal axis starts from the sizing of the rotor, so its power $[P]$ (Equation 1) is calculated as a function of the fluid's density $[\rho]$, the area swept by the blade of the rotor ( $A=\pi R^{2}$, where $\mathrm{R}$ is the radius of the turbine), the fluid's speed $[V]$, the power coefficient $\left[C_{p}\right]$ and the efficiency of the mechanism's drive train $[\eta]$ (gears, generator, and others), being considered in this work for the latter a value of $70 \%$, according to the literature ${ }^{8}$.

$$
P=\frac{1}{2} \rho \pi R^{2} V^{3} C_{P} \eta
$$

The performance of the hydrokinetic turbine is characterized by its power coefficient $\left[C_{p}\right]$ (Equation 2), that represents the relation between the extracted power of the current of water and the available power in the current that goes through the area projected by the turbine. This implies that the turbine cannot fully extract the contained energy in the water flow, so the limit established by Betz for this $C_{p}$ is 0.593 . 


$$
C_{p}=\frac{P}{0.5 \rho \pi R^{2} V^{3}}
$$

This coefficient depends at the same time of the TSR $[\lambda]$ (Tip Speed Relation). For this reason, the existing relation between the speed of the blade in the tip and the water current, has a big influence in the turbine's efficiency, being a fundamental factor that must be maximized to obtain the best possible power coefficient and optimize the extraction of the contained energy in these currents ${ }^{8}$. This relation is defined through Equation 3.

$$
\lambda=\frac{R \omega}{V}
$$

\section{Methodology}

The development of the three-dimensional models of the hydrokinetic turbine of horizontal axis of first, second and third generation, with their respective fluid volumes, were carried out using the commercial program NX10.0 of Siemens. The three models are composed of a rotating volume and a stationary volume Figure 2 , which were established according to the work done by $\frac{8-10}{}$.

The first model, known as the first-generation hydrokinetic turbine Figure 3a, has a radius of $0.75 \mathrm{~m}$ and consists of an axis (also known as a cube) and three blades, which were designed using the hydrodynamic profile NREL S822, selected by its self-cleaning properties ${ }^{8}$ and an angle of attack of $5^{\circ}$. A geometric simplification is also made to a third of the model, taking advantage of its geometric and dynamic symmetry, to reduce computational costs. The first-generation hydrokinetic turbines, as well as the fluid volume dimensions, are presented in Figure 3, where $\mathrm{R}$ denotes the radius of the turbine.

For the elaboration of the external fluid volume, it was ensured that the wall effects do not affect the hydrodynamic profile of the fluid, for which reason a distance of $1 \mathrm{R}$ was established in the longitudinal direction upstream of the turbine (Figure $3 \mathrm{~b}$ ), according to what was established by 11 and $0.5 \mathrm{R}$ radially as shown in Figure 4.

The second model, denoted as a second-generation hydrokinetic turbine, is composed of the same turbine of the first-generation model, incorporating on it a profiled diffuser (Figure 5a), which was designed using the hydrodynamic profile NREL S822. The stationary volume (external fluid) of this model has a cylindrical shape, while the rotating volume (internal fluid) conforms to the internal shape of the diffuser. The dimensions of the fluid volumes are shown in Figure 5b and have higher values than those used in the first generation model, because the diffuser covers a larger area, besides considering that under the same flow conditions, the profile of the boundary layer presented at the outlet of the diffuser, is farther from the axis of the turbine, which is why a stationary volume with greater proportions is necessary, guaranteeing as it is recommended in the literature, that the wall effects do not affect the flow.

The third and last model, known as the thirdgeneration hydrokinetic turbine, is formed, in addition to the turbine of the first-generation model, by two coupled devices Figure 6a. The first one is a profiled enclosure that surrounds the rotor, which also fulfills the role of a first diffuser, which was developed from the design parameters of the diffuser used in the second-generation model, to analyze its performance in this new configuration. The second mechanism is a divergent diffuser that is coupled to the outlet of the housing. The volume of stationary fluid (external fluid) has a cylindrical shape, while the rotary fluid adopts the same shape as the internal part of the housing and the diffuser. These dimensions, for the reasons mentioned in the previous model, have higher values than those used for the first and second generation hydrokinetic turbines. The dimensions of the fluid volume where the third-generation turbine is submerged are presented in Figure $6 \mathrm{~b}$.

The fluid volumes of the first, second and third generation hydrokinetic turbine were exported to the commercial program ANSYS V18.2, in which the domains and their regions of interest were defined and the respective meshing process was performed. For the three models, unstructured meshes formed mainly by tetrahedral elements were used, restructuring the mesh size to $1 \mathrm{~mm}$ on the trailing edge of the blade profile. This was to satisfy the $\mathrm{Y}+$ requirement of the turbulence model used, in consideration of the release of the boundary layer and instabilities in the fluid in this zone. For the first-generation turbine, a proximity and curvature algorithm was used, with a minimum mesh size of $5 \mathrm{~mm}$, a maximum face size of $10 \mathrm{~mm}$ and a maximum size of tetrahedron of $20 \mathrm{~mm}$. For the second-generation turbine, a curvature algorithm was applied, with a minimum mesh size of $35 \mathrm{~mm}$, a maximum face of $45 \mathrm{~mm}$ and a maximum tetrahedron size of $55 \mathrm{~mm}$. For the thirdgeneration hydrokinetic turbine, a curvature algorithm was implemented, with a minimum mesh size of $40 \mathrm{~mm}$, a maximum face size of $50 \mathrm{~mm}$ and a maximum tetrahe- 
dron size of $60 \mathrm{~mm}$. These values were established from the mesh independence study performed for each model. The number of mesh nodes used was 3.66E + 06, 1.76E + 06 and 3.62E + 06, for the first, second and third generation hydrokinetic turbine, respectively.

Subsequently, the models were analyzed using a CFD analysis (Computational Fluid Dynamics), in the CFX module of the ANSYS V18.2 program. The three computational models were configured in a transitory regime characterized by a total time of $4 \mathrm{~s}$ for the first-generation turbine and $6 \mathrm{~s}$ for the second and third generation mechanisms, seeking stability in the behavior of the fluid. A step time of 1.0E-02 $\mathrm{s}$ was used for all cases, guaranteeing maximum RMS values of $1.0 \mathrm{E}-04$ in the conservation of mass and momentum as the convergence criterion. Torque monitors were established in the turbine shaft to determine the stability in its behavior, from the quantification of its temporal variation and the verification of the transition from steady to stationary state in the operation of the turbine, to guarantee a fully developed flow. Water at room temperature was used as the working fluid, a turbulence model $\mathrm{k}-\varepsilon$, according to the studies carried out by ${ }^{3.8}$, the interface between the walls of the stationary and the rotary volume was configured as "frozen rotor" based on the study carried out by $\underline{12}$. A double precision study was set to reduce numerical errors.

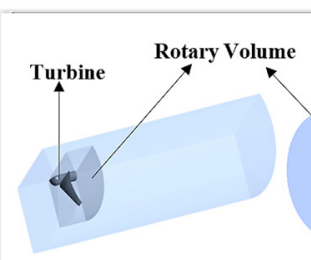

a)

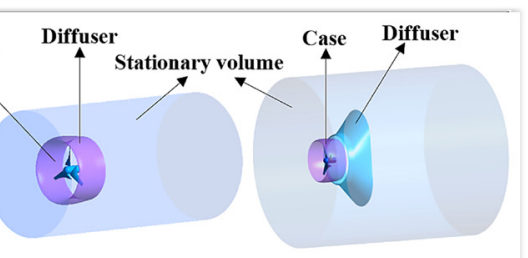

b)

c)
Figure 2. Fluid volume of the horizontal axis hydrokinetic turbine: (a) first generation, (b) second generation and (c) third generation diffuser. Own source.

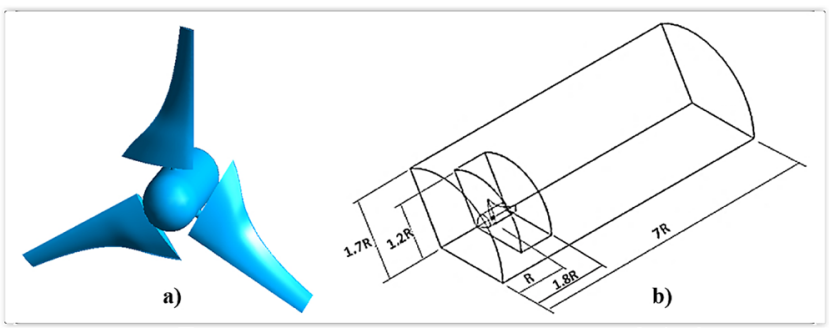

Figure 3. First generation hydrokinetic turbine:(a) complete model of the turbine and (b) geometric simplification to one third of the model within its fluid volumes, dimensioned according to the radius $[R]$ of the turbine. Own source.

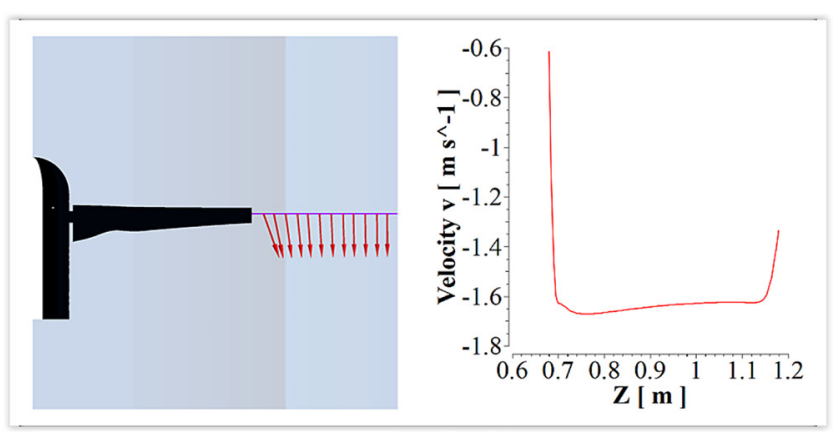

Figure 4. Incidence of wall effects in the hydrodynamic profile of the turbine blade. Own source,

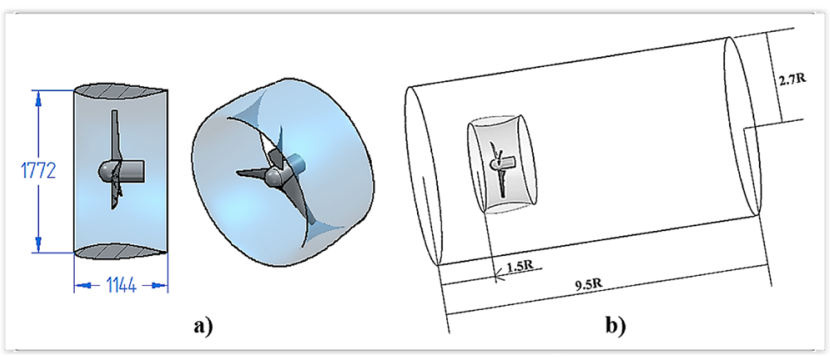

Figure 5. Second generation hydrokinetic turbine: (a) diffuser, with its dimensions [mm] and (b) within the fluid volumes, sized according to the radius $[R]$ of the turbine. Own source.

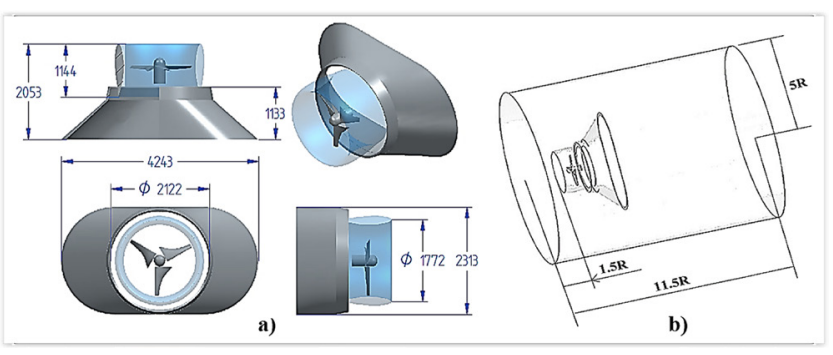

Figure 6. Third-generation hydrokinetic turbine: (a) housing and diffuser, with their dimensions $[\mathrm{mm}]$ and (b) within the fluid volumes, sized according to the radius [R] of the turbine. Own source.

\section{Results and Discussions}

Figure 7 shows the Power Coefficient $\left[C_{p}\right]$ of the first, second and third generation hydrokinetic turbine, as a function of the TSR $[\lambda]$ (Tip Speed Ratio), with a TSR interval between 0 and 160 with steps of 5 . These results, represented by point curves, show a parabolic behavior of $C_{p}$ with respect to the variation of the TSR, which is consistent with the numerical and experimental result found in the literature for this type of turbines ${ }^{11,13}$. The maximum $C_{p}$ reached by the turbine of 1 st, 2 nd and 3 rd 


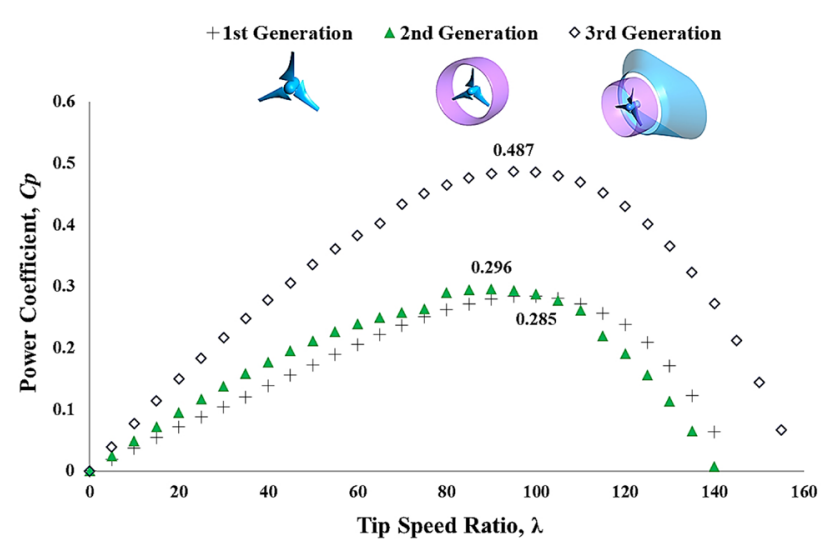

Figure 7. Power Coefficient $\left[C_{p}\right]$ as a function of the Point Speed Ratio $[\lambda]$ of the first, second and third generation horizontal axis hydrokinetic turbine blade. Own source.

generation is $0.285,0.296$ and 0.487 , at a TSR of 100 , 90 and 95, respectively. The second-generation turbine presents an increase in $C_{p}$ of $3.7 \%$ compared to the firstgeneration model, while the Third-generation turbine shows an increase of $41.5 \%$ and $39.2 \%$ with respect to the first and second generation. This represents an efficiency of $82.1 \%$ of the latest generation turbine compared to that established by the Betz limit.

Figure 8 shows the contours and velocity vectors in the mean transverse section of the first, second and third generation turbine blade (Figure 8a, Figure $8 \mathrm{~b}$ and Figure $8 \mathrm{c}$, respectively), at a distance of $0.34 \mathrm{~m}$ measured from the tip of the blade. The color scale of the velocity profile of the three models comprises values between 0 and $2.6 \mathrm{~m} \mathrm{~s}^{-1}$. In all three cases, the speed presented upstream of the turbine is higher than the speed that is downstream of it, while the greater acceleration of the fluid is generated in the lateral zones external to the entrance and outlet edges of the hydrodynamic profile, especially, downstream of this. There is also a release of the boundary layer in the profile of the turbine blade, because of the high inlet velocity of water. On the other hand, the velocity vectors show a normal behavior of the flow in the areas where there is no direct obstruction to the passage of water by the blade, while in the middle region downstream of this, where partial interruption occurs of the fluid, a recirculation phenomenon is generated. These re-circulations, as well as the detachment of the boundary layer, produce energy losses in these mechanisms.

Figure 8 shows an increase in the speed at the inlet of the third-generation turbine (Figure 8c) with speeds between 1.5 and $2.1 \mathrm{~ms}^{-1}$, while the turbine of first and second generation (Figure 8a and Figure 8b, respectively) have lower values in the same areas with speeds ranging between 1.5 and $1.8 \mathrm{~ms}^{-1}$. There is evidence for all cases indicating a decrease in the velocity of the fluid both in the upper and lower regions where the profile of the turbine blade is located, which is consistent given the obstruction that is generated by the passage of water. There is also an increase in speed downstream of the blade, in areas where there is no direct impact of this, where the speed is more homogeneous in the second and third generation turbine, presenting higher speeds in the latter model with values between 2.3 and $2.6 \mathrm{~ms}^{-1}$, with respect to the turbine of first and second generation that reach values that oscillate between 1.8 and $2.1 \mathrm{~ms}^{-1}$. The speeds downstream of the profile show a greater stagnation of water for the first-generation turbine, in addition to presenting more homogeneous and concentrated areas with values between 0 and $0.5 \mathrm{~ms}^{-1}$, of which can be seen that the area of greatest stagnation, where speeds have zero, takes the form of a bell. While, the second and third generation turbine present very similar results to each other, with speeds ranging between 0 and $0.8 \mathrm{~ms}^{-1}$, being in both cases that the lower speeds and therefore the greater stagnation of water is presented in the area of exit of the diffuser that surrounds the turbine, where it can be observed also that this region takes the form of an inverted bell, similar to that presented by the first generation mechanism, generating that the stagnant water originates in greater proportion at a greater distance from the profile of the blade and consequently, the losses are smaller with respect to the first model.

Figure 9 shows the contours and velocity vectors $\left[\mathrm{m} \mathrm{s}^{-1}\right]$ in the average cross section of the second and third generation turbine model (Figure 9a and Figure 9b, respectively). These models were selected to carry out the comparison, for presenting a higher power coefficient with respect to the first-generation turbine, in addition to the geometric similarities between both, by sharing the design of the diffuser, also called casing in the model of the thirdgeneration turbine. The velocity profiles of both models comprise a scale of values between 0 and $2.6 \mathrm{~m} \mathrm{~s}^{-1}$, where the velocity presented upstream of the turbine is higher than that found downstream of it and the greater acceleration of the fluid is generated in the external lateral zones of the inlet and outlet edges of the hydrodynamic profile, especially downstream of this. Figure $9 \mathrm{~b}$ shows that the opening between the casing and the diffuser (area of 
interest) allows the external fluid to enter the mechanism, increasing the speed of the water inside it downstream, from $1.5 \mathrm{~ms}^{-1}$ to $2.1 \mathrm{~ms}^{-1}$, reducing the recirculation of fluid at the trailing edge of the casing profile, also helping to have greater control and better performance by avoiding the release of boundary layer in this area, as proposed in the study of $\underline{\underline{5}}$. This increase in the speed of the fluid provides a greater thrust of the flow downstream of the third-generation turbine with respect to the secondgeneration model, allowing evacuating the water held there or re-circulating. While the second-generation turbine (Figure 9a), presents in the same area of interest, speeds between 1.5 and $1.8 \mathrm{~m} \mathrm{~s}^{-1}$.

There are not enough studies in the literature related to the implementation of third generation diffusers in hydrokinetic turbines, which are necessary to perform a qualitative validation of the results obtained. The study developed by ${ }^{\underline{Z}}$ shows a great similarity with respect to the objective of the present work, which propose a similar mechanism, that differs basically in the use of a third diffuser downstream of the turbine of the model in comparison. The mechanism proposed by the authors, with their respective components, is presented in Figure 10.

In their study ${ }^{7}$ used a fluid input velocity of $1.2 \mathrm{~m} \mathrm{~s}^{-1}$, which increased until reaching average speeds of $1.9 \mathrm{~m} \mathrm{~s}^{-1}$ downstream of the turbine by implementing the first convergent diffuser-divergent (1), equivalent to an increase in speed of $36.8 \%$. This improvement is similar to the second-generation turbine (Figure $5 \mathrm{a}$ ) proposed in this work, with which, based on the average fluid velocity of $1.5 \mathrm{~m} \mathrm{~s}^{-1}$, average speeds of $1.9 \mathrm{~m} \mathrm{~s}^{-1}$ were obtained in this same area, representing an increase in speed of $21.1 \%$.
This shows that the model proposed by ${ }^{7}$ exceeds by $42.7 \%$ the average speed produced compared to the secondgeneration turbine. This may be due to the geometry of the diffuser, especially when having a completely circular throat, as presented by ${ }^{3}$, which propose a diffuser with a completely circular throat and a divergent exit, finding an increase of $310 \%$ of the power generated by the turbine, while the second-generation turbine proposed in this study showed a $4 \%$ increase in the power generated by the turbine without this device.

The improvements (2) and (3) raised by ${ }^{7}$ for its increased diffuser, allowed him to obtain average speeds downstream of the turbine $2.6 \mathrm{~ms}^{-1}$, equivalent to an increase in the speed of $58.3 \%$ in relation to the initial fluid velocity of $1.2 \mathrm{~ms}^{-1}$. This improvement is similar to the third-generation turbine (Figure 6a) proposed in this study, with which average speeds of $2.5 \mathrm{~ms}^{-1}$ were reached downstream of the turbine, representing an increase in speed of $40.0 \%$ with respect to the initial fluid velocity of $1.5 \mathrm{~ms}^{-1}$. Therefore, the increase presented in the speed by the model proposed by ${ }^{\mathrm{z}}$ is $31.4 \%$ higher than that generated by the third-generation hydrokinetic turbine. The difference in the speed increases found in this work and in the mechanism proposed $^{7}$ can be linked mainly to two factors, the first one based on the geometric differences that the diffusers of both models present and the second is about not taking into account the turbine at the moment of hydro dynamically analyzing the device proposed by ${ }^{\mathrm{T}}$, which does not quantify the loss of energy that comes with the interaction between the fluid and the rotor. An important factor to increase the efficiency of this kind of turbines is shown in this study; to increase the speed downstream of the rotor, to reduce the areas of stagnation

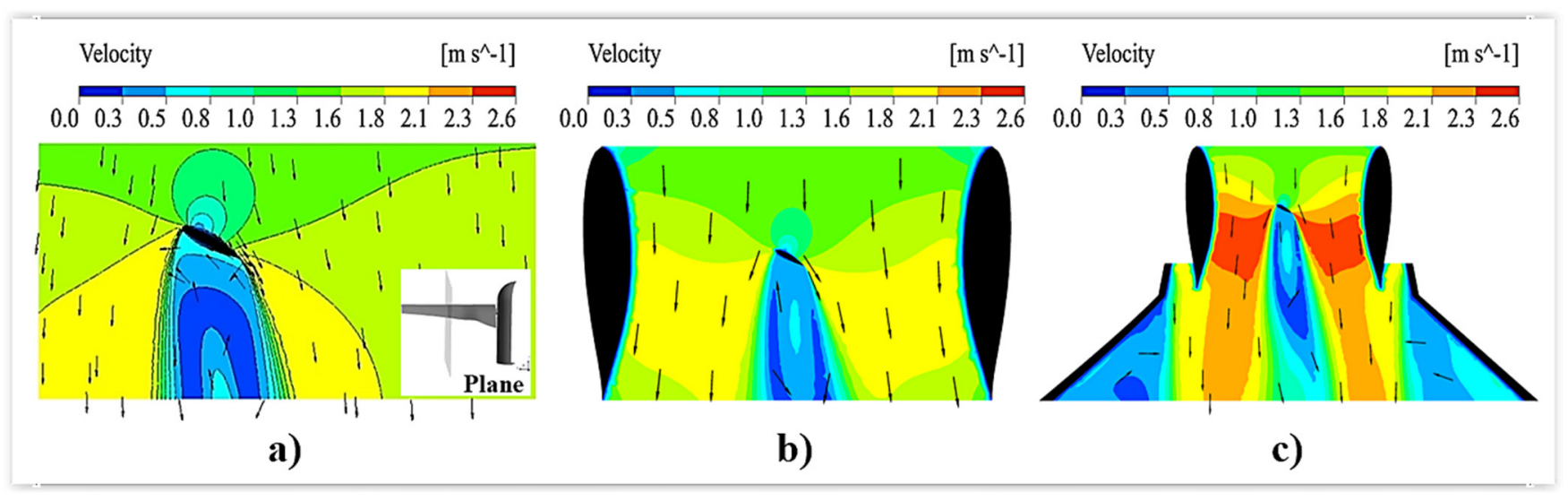

Figure 8. Contour and velocity vectors $\left[\mathrm{m} \mathrm{s}^{-1}\right]$ in the mean cross section of the turbine blade of: a) first, b) second and c) third generation. Own source. 


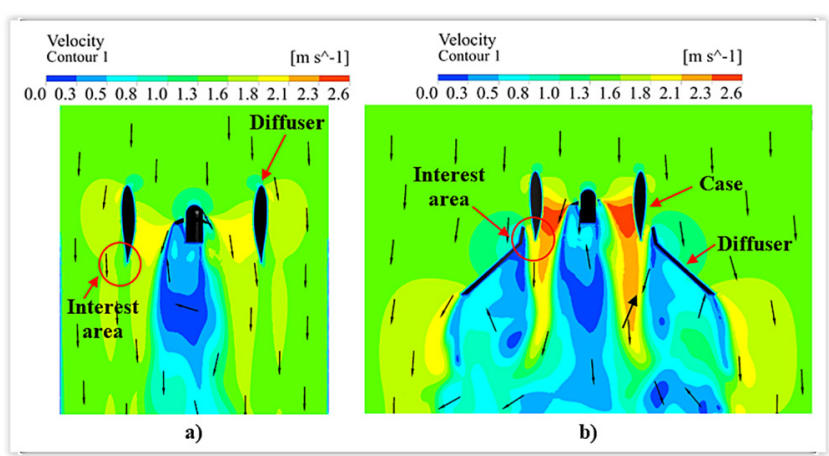

Figure 9. Contour and velocity vectors $\left[\mathrm{m} \mathrm{s}^{-1}\right]$ in the average cross section of the turbine model of: a) second and b) third generation. Own source.

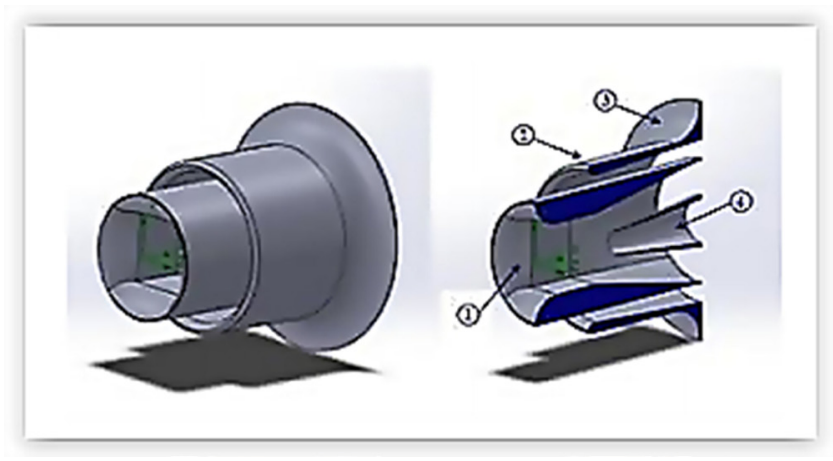

Figure 10. Section view of the final configuration. (1) Main Venturi nozzle - (2) Secondary diffuser R - (3) Flange S (4) Internal diffuser $\mathrm{DDi}^{7}$.

and recirculation of the fluid, also demonstrating that the integration of two or more diffusers is a very promising option, allowing in both cases to increase the power coefficient $[\mathrm{Cp}]$ of hydrokinetic turbines with values higher than 0.4 .

\section{Conclusions}

Of the analyzed models, the third-generation hydrokinetic turbine shows a better performance and a great superiority, presenting an increase in the Cp of $41.5 \%$ and $39.2 \%$ with respect to the turbine of first and second generation, respectively. Representing an efficiency of the turbine of $82.1 \%$ in comparison to that established by the Betz limit, in turn agrees with that stipulated where they state that this type of mechanism can achieve efficiency close to $90 \%$.

The second-generation turbine has an increase in the $\mathrm{Cp}$ of $3.7 \%$ compared to the first-generation model, which shows that the implementation of diffusers allows increasing the efficiency of hydrokinetic turbines. Despite this, the increase is not significant if it is compared with the results obtained from the third-generation turbine, considering that this device was used as a casing, reflecting that there is a wide field of research with respect to various improvements or modifications that could be made on this type of mechanisms.

The efficiency of hydrokinetic turbines can increase greatly with the acceleration of the fluid downstream of them, finding that the implementation of third-generation mechanisms, where the integration of various diffusers is promoted, fulfills the function of a speed multiplier in these zones, reaching promising results. The thirdgeneration turbine provides greater use of the available water resources, reaching speeds ranging from 1.5 to $2.1 \mathrm{~m} \mathrm{~s}^{-1}$, and 2.3 to $2.6 \mathrm{~m} \mathrm{~s}^{-1}$ upstream and downstream of the turbine, respectively. Finding that the opening between the casing and the diffuser allows the external fluid to enter the mechanism, inducing an increase in the speed downstream of the turbine from 1.5 to $2.1 \mathrm{~m} \mathrm{~s}^{-1}$, representing an increase in speed of $28.6 \%$, with which the stagnation and the recirculation of the fluid in this zone is diminished, and in turn reflected in the decrease of energy losses of the model.

\section{References}

1. Badea I, Pricop MV, Bobonea A. Design procedure and numerical analysis of a small horizontal - axis hydrokinetic turbine. UPB Scientific Bulletin, Series D: Mechanical Engineering. 2014; 76(3):1-10.

2. Filho GLT, Souza Z De, Rossi C a B De, Barros RM, Silva FDGB Da. Poraque hydrokinetic turbine. IOP Conference Series Earth and Environmental Science. 2010; 12(1):12094.

3. Gaden DLF, Bibeau EL. A numerical investigation into the effect of diffusers on the performance of hydro kinetic turbines using a validated momentum source turbine model. Renewable Energy. 2010 Jun; 35(6):1152-8. https://doi.org/ 10.1016/j.renene.2009.11.023

4. Yuce MI, Muratoglu A. Hydrokinetic energy conversion systems: A technology status review. Journal of Renewable and Sustainable Energy. 2015; 43:72-82.

5. Els RH Van, Junior ACPB. The Brazilian experience with hydrokinetic turbines. Energy Procedia. 2015; 75:259-64. https://doi.org/10.1016/j.egypro.2015.07.328

6. Khan MJ, Bhuyan G, Iqbal MT, Quaicoe JE. Hydrokinetic energy conversion systems and assessment of horizontal and vertical axis turbines for river and tidal applications: A technology status review. Applied Energy. 2009; 86(10): 1823-35. https://doi.org/10.1016/j.apenergy.2009.02.017 
7. Piancastelli L, Clarke RV, Cassani S. Diffuser augmented runs the river and tidal pico-hydropower generation system. ARPN Journal of Engineering and Applied Sciences. 2017; 12(8):2678-88.

8. Chica E, Perez F, Rubio-Clemente A, Agudelo S. Design of a hydrokinetic turbine. WIT Transactions on Ecology and the Environment. 2015; 195:137-48. https://doi.org/10.2495/ ESUS150121

9. Schleicher WC, Riglin JD, Kraybill Z a, Gardner G. Design and simulation of a micro hydrokinetic turbine. Proceedings of the 1st Marine Energy Technology Symposium METS13; 2013.

10. Subhra Mukherji S, Kolekar N, Banerjee A, Mishra R. Numerical investigation and evaluation of optimum hydrodynamic performance of a horizontal axis hydrokinetic turbine. Journal of Renewable and Sustainable Energy. 2011; 3(6):63105. https://doi.org/10.1063/1.3662100
11. Kolekar N, Banerjee A. Performance characterization and placement of a marine hydrokinetic turbine in a tidal channel under boundary proximity and blockage effects. Applied Energy. 2015; 148:121-33. https://doi.org/10.1016/ j.apenergy.2015.03.052

12. Kim SJ, Singh PM, Hyun BS, Lee YH, Choi YD. A study on the floating bridge type horizontal axis tidal current turbine for energy independent islands in Korea. Renewable Energy. 2017; 112:35-43. https://doi.org/10.1016/j.renene. 2017.05.025

13. Schleicher WC, Riglin JD, Oztekin A. Numerical characterization of a preliminary portable micro-hydrokinetic turbine rotor design. Renewable Energy. 2015; 76:234-41. https://doi.org/10.1016/j.renene.2014.11.032 